\title{
Experimental evaluation of UAV spraying for peach trees of different shapes: effects of operational parameters on droplet distribution
}

\author{
Yanhua Meng ${ }^{\mathrm{a}, \mathrm{b}, \mathrm{c}}$, Jinya Su${ }^{\mathrm{d}}$, Jianli Song ${ }^{\mathrm{e}, *}$, Wen-Hua Chen ${ }^{\mathrm{f}}$, Yubin Lan ${ }^{\mathrm{a}, \mathrm{b}, *}$ \\ ${ }^{a}$ College of Electrical Engineering, South China Agricultural University, Guangzhou, 510642, China \\ ${ }^{b}$ National Center for International Collaboration Research on Precision Agricultural Aviation Pesticide Spraying Technology, \\ Guangzhou, 510642, China \\ ${ }^{c}$ Key Laboratory of Aviation Plant Protection, Ministry of Agriculture and Rural Affairs, Anyang, 455000, China \\ ${ }^{d}$ School of Computer Science and Electronic Engineering, University of Essex, Colchester, CO4 3SQ, U.K. \\ ${ }^{e}$ College of Science, China Agriculture University, Beijing, 100193, China \\ ${ }^{f}$ Department of Aeronautical and Automotive Engineering, Loughborough University, Loughborough, LE11 3TU, U.K.
}

\begin{abstract}
Small-scale plant protection Unmanned Aerial Vehicles (UAVs) are finding a wide range of applications in modern agriculture management (including aerial spraying) due to their high efficiency and flexibility, low labour/water requirement and no damage to crops and soils, which substantially increase agricultural productivity and sustainability.
\end{abstract} UAV operational parameters, however, have remarkable effects on droplet distribution in UAV spraying, which significantly affect pesticide utilization rate and treatment effectiveness. Therefore, this work aimed to evaluate the effects of UAV operational parameters on droplet distribution for orchard trees. In particular, peach, an important orchard tree worldwide, is investigated in this study, and two typical tree shapes were considered including Y-shape and Central Leader (CL)-shape. Specifically, UAV spraying experiments were performed in Shandong Institute of Pomology, Shandong Province, China, and gas powered helicopter 3WQF120-12 was chosen as the spraying platform. The UAV operational parameters under consideration include flight route (intra-row, inter-row), flight velocity (four levels: 2,3 , $4,5 \mathrm{~m} / \mathrm{s}$ ), number of spray times (1 vs 2) and nozzle flow rate. Droplet coverage rate at different positions and layers, obtained by water sensitive papers, was chosen as the metric to evaluate spraying performance. Experimental results show that: (1) the spraying uniformity is different between Y-shape and CL-shape peach tree, where Y-shape exhibits uniformity for positions at inner or outer layers. CL-shape results in a higher droplet coverage at top layer while with uniformity at lower three layers; (2) for Y-shape peach, intra-row route obtained a higher droplet coverage rate; while for CL-shape peach inter-row not only saved spraying volume but also results in a higher droplet coverage rate; (3) for both tree shapes, the increase in flight velocity $(2-5 \mathrm{~m} / \mathrm{s})$ significantly decreased the droplet coverage rate; (4)

\footnotetext{
* Jianli Song and Yubin Lan are corresponding authors; Yanhua Meng and Jinya Su are joint first authors contributing equally to this work.

Email addresses: Yanhua.Meng@outlook.com (Yanhua Meng), j.su@essex.ac.uk (Jinya Su), songjianli170@163.com (Jianli Song), W.Chen@lboro.ac.uk (Wen-Hua Chen), ylan@scau.edu.cn (Yubin Lan)
} 
for Y-shape peach with doubling the number of spraying times decreased the spraying performance for unit area. (5) for CL-shape peach with intra-row route, increasing the nozzle flow rate from 1.8 to $2.2 \mathrm{~L} \cdot \mathrm{min}^{-1}$ can significantly improve the droplet coverage rate at top and bottom two layers. It is envisioned that this study can provide some fundamental guidance of the operation of small UAVs for the aerial spraying of peach trees and similar orchards. Keywords:

Droplet coverage; Operational parameters; Peach trees; Plant protection; Unmanned Aerial Vehicle (UAV).

\section{Introduction}

Plant pests, weeds and diseases are significantly affecting agricultural production and threatening food security worldwide [1. It is estimated that about $30 \%$ of the crop loss worldwide is due to the adverse effects of weeds, diseases and insect pests annually [2. This problem is even more severe in consideration of population growth/ageing, resource shrinkage, environment and climate changes [3, 4. Therefore, it is of paramount importance to adopt an efficient crop protection system to prevent and control plant diseases and pests, increasing agricultural productivity and sustainability concurrently. At present, chemical spraying still remains the most effective method for pest/disease prevention and control worldwide [5]. In this regard, there are various spraying manners with different efficiencies in practice including manual backpack, ground mechanical spraying, manned/unmanned aerial spraying [6].

Aerial spraying by small-scale Unmanned Aerial Vehicles (UAVs) is an emerging practice in many countries and regions including China [7. This approach possesses a number of advantages over conventional strategies. In particular, in comparison to manual backpack and ground mechanical approaches: firstly, it is less labour-intensive and poses no hazardous threat to human health; secondly, it is less reliant on crop types (e.g. high-stalk crops, paddy fields) and environmental conditions (e.g. mountainous terrains, muddy fields); thirdly, it is more efficient in term of pesticide utilization rate (since on the one hand UAVs adopts ultra-low volume spraying and on the other hand downwash airflow generated by UAV rotors increases penetrability of droplets on crops [8]). While, in comparison to manned aerial spraying, UAV spraying is suitable for field-scale (i.e. small to medium scale) farms or orchards, requiring no specific take-off and landing sites [2].

In UAV spraying, droplet distribution on target canopy is one of the paramount metrics for evaluating spraying performance. Inappropriate droplet deposition usually results in pesticide waste, control effect reduction or even pollution and poisoning. In practical applications, however, a number of factors affect droplet distribution, such as aerial nozzle type, droplet size, operational parameters of plant protection UAVs (e.g. flight height, flight velocity) [5]. 
A lot of conventional studies were mainly focused on evaluating the effects of aerial nozzle type [9], droplet size [10], nozzle pressure and inclination on droplet deposition. With the widespread application of plant protection UAVs, the evaluation of UAV operational parameters has now become a hot research topic in recent years.

It is noted that most of the existing results are on cereal crops rather than orchard trees. For example, the effects of flight height and flight velocity on droplet deposition distribution were investigated in wheat [1], cotton [12] and indoor environment [13, respectively. It was shown in both crops [11] 12] that increase of flight height within a certain range (i.e. with appropriate degree of downwash airflow) could improve the droplet deposition density and coverage rate. The functional relationship between droplet distribution characteristics and flight height and velocity for a small helicopter was investigated in wheat [14] and rice [15, respectively. The effects of spraying volume and nozzle size on droplet deposition and disease control for wheat crop [16] was investigated. It was shown that coarser nozzle at $>16.8 \mathrm{~L} \cdot h \mathrm{a}^{-1}$ volume resulted in the best control efficacy. The effects of flight mode (forward and backward) and environmental crosswind were tested in [17] by using the method of deposition quality balance. It was shown that the environmental crosswind had a significant impact on droplet deposition distribution. The downwash flow field of the rotors also has effects on droplet movement and deposition, which was investigated in rice [2], indoor environment [18] and rice field [19] for multicopters and helicopters, respectively. There are also a few studies on orchard trees, although the number is very limited in comparison to cereal crops. In particular, the effects of flight height, velocity, and nozzle flow rate for a six-rotor UAV were investigated on citrus canopy [20, 21]. It was shown that the factors affecting droplet deposition density and penetrability were both in the order of flight velocity, height and nozzle flow rate 20. Recently, in addition to operational height $(0.6 \mathrm{~m}, 1.2 \mathrm{~m}$, and $1.8 \mathrm{~m})$, the effects of tree shape (triangle-shape and inverted triangle-shape) were investigated for citrus tree in 22, where tree shape was shown to have significant effects on droplet deposition at specific layers.

On the other hand, peach (Prunus persica) is originated in China and is now an important fruit tree worldwide. According to the Food and Agriculture Organization (FAO), as of 2010, peach production in China accounts for $50 \%$ of the world production. However, peach tree is subject to a number of pests and diseases (e.g. peach twig borer, oriental fruit moth, summer fruit totrix moth) at its different growing stages [23, affecting both quantity and quality of peach production. The commonly used approaches of preventing and controlling pests and diseases are to spray pesticides and fungicides [24]. Manual backpack spraying by human being is feasible (although inefficient) for Central Leader (CL)-shape peach tree, since there is enough spacing between tree rows. However, this approach brings difficulties for Y-shape peach tree due to a lack of row spacing (please refer to Fig 2 for row spacing of different tree shapes). More 
importantly, human spraying is labour-intensive, inefficient and poses hazardous threats to human health. In this regard, small-scale plant protection UAVs have many potentials due to their high efficiency and flexibility, and low labour/water requirement. However, UAV spraying performance should be assessed and UAV operational parameters should also be optimized before their potentials being fully realized, which is the primary objective of this study. To summarize, the motivation of this study was to highlight the research gap with the following observations:

(1) Small-scale plant protection UAVs possess a number of advantages over conventional pesticide spraying strategies, however, their performance should be evaluated and their operational parameters should be optimized before being commercialized for different applications.

(2) A lot of studies are performed on the effects of UAV operational parameters on droplet distribution for cereal crops (e.g. wheat, paddy, maize), however, little attention has paid to the study of orchard tree which is the most demanding area of research in recent time.

(3) A number of operational parameters (e.g. flight height, flight velocity, flight pattern) are investigated in previous studies for cereal crops. However, few studies have focused on orchard trees. In addition, flight route, number of spray times and tree shape are to be investigated for orchard trees, either.

(4) Peach is an important orchard tree worldwide, however, its operational parameters for aerial spraying have not been investigated, letting alone for various types of tree shape.

Therefore, the aim of the present study was to experimentally evaluate the effects of UAV operational parameters on droplet coverage rate [8] for peach trees, so that commercial UAV spraying system can thus be developed. In particular, two typical peach tree shapes were considered in this study including Y-shape and CL-shape. The considered operational parameters included flight route (intra-row, inter-row), flight velocity (four levels: 2, 3, 4, 5 m/s), number of spray times (1 vs 2) given spray volume per unit area and nozzle flow rate $\left(1.8\right.$ vs $\left.2.2 \mathrm{~L} \cdot \mathrm{min}^{-1}\right)$. Gas powered helicopter 3WQF120-12 was chosen as the platform for UAV spraying experiments. Droplet coverage rate, obtained by water sensitive papers, was chosen as the metric to evaluate spraying performance. It is expected that this study can provide some fundamental and practical guidances for UAV spraying of peach trees and related orchard trees with a similar structure. The remainder of this paper is organized as follows: the materials and methods section describe the detail of experiment site, peach tree types, small scale plant protection UAV, experiment droplet coverage evaluation and data analysis; the main results along with discussions are given in Section 3 Section 4 concludes the paper with 
future work discussions.

\section{Materials and methods}

In this section, materials and methods relevant to the study are introduced such as the experimental site, peach trees, small-scale plant protection UAV, experiment deign, droplet coverage evaluation and data analysis.

\subsection{Experimental site and peach trees}

Field experiments were conducted in the experimental orchard (geographic coordinate information with latitude: $36^{\circ} 219^{\prime} N$, longitude: $117^{\circ} 020^{\prime} E$ and altitude: $167 \mathrm{~m}$ a.s.l.) of Shandong Institute of Pomology, Shandong Province, China (please refer to Fig 1). The region is of temperate continental subtropical monsoon climate, where the mean annual temperature, rainfall and hours of sunshine are 12.9C, 697mm (mostly in June-September) and 2627h, respectively. The experiments were conducted on 27/July/2019 with fully developed peach leaves in size. Peach variety of Chaohong was adopted in this study. Two typical shapes of peach tree were investigated including Y-shape and CL-shape, where their spatial characteristics are displayed in Fig 2, As displayed in Fig 4 and Fig 5 , the tree height, row spacing, tree distance in a row for Y-shape and CL-shape peach trees are $3.5 \mathrm{~m}, 4 \mathrm{~m}, 2 \mathrm{~m}$, and $3 \mathrm{~m}, 4 \mathrm{~m}, 1.5 \mathrm{~m}$, respectively.

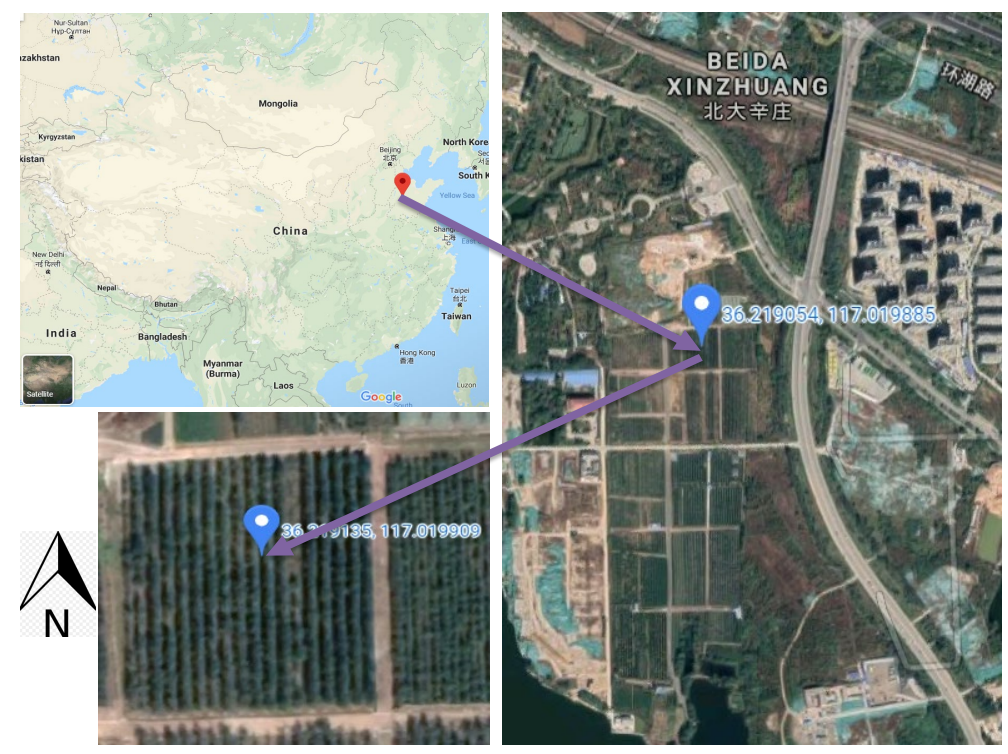

Figure 1: Geographic location of the experimental orchard in this study. 


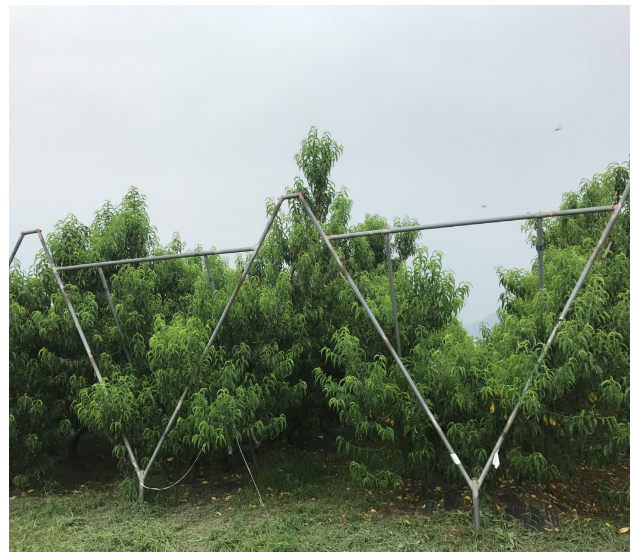

Y-shape peach tree

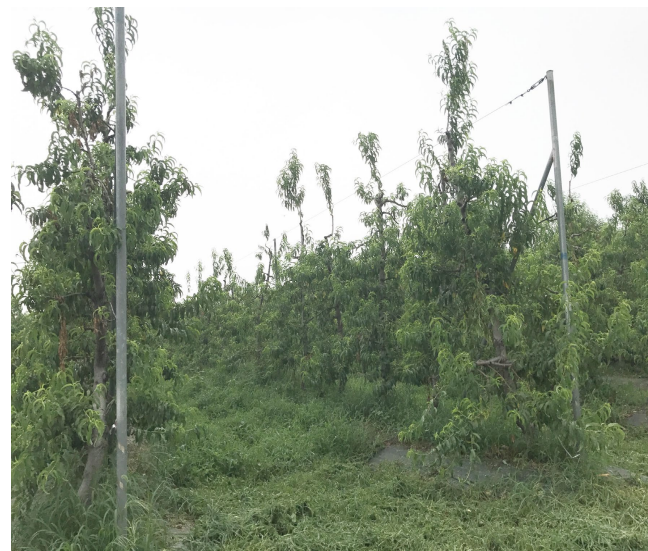

CL-shape peach tree

Figure 2: Images of Y-shape (left) and CL-shape (right) peach trees in the present study.

\subsection{Plant protection $\boldsymbol{U A V}$}

In this study, gas powered helicopter 3WQF120-12 (Anyang Quanfeng Biological Technology Co., Ltd., China) is adopted as the small-scale plant protection UAV (please refer to Fig 3 for the UAV spraying on V-shape peach), which has also been applied in a number of previous studies [5, 25]. The specification and flight parameters of the UAV in

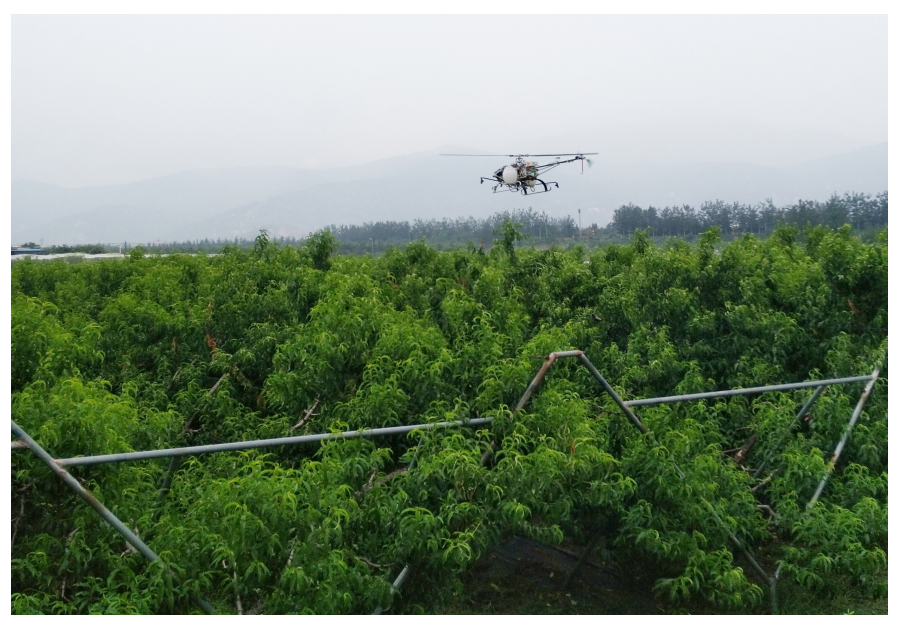

Figure 3: Image for the plant protection UAV in this study: spraying on V-shape peach tree.

this study are summarized in Table 1

\subsection{Experiment design}

Following the volume adjustment standards for UAV spraying [8], given the total nozzle flow rates $R_{\text {nozzle }}$ and spraying swath $L_{\text {swath }}$, spray volume per unit area $V_{\text {volume }}$ is a function of flight velocity $V$, given by formula (1)

$$
V_{\text {volume }}=\frac{R_{\text {nozzle }}}{V \times L_{\text {swath }}} .
$$


Table 1: Physical and flight parameters of plant protection UAV 3WQF120-12.

\begin{tabular}{c|c||c|c}
\hline Name & Value & Name & Value \\
\hline No. of Rotor & 1 & Flight velocity $/ m \times s^{-1}$ & $2-5$ \\
\hline Nozzle type & Flat-fan, Lu120-015 & Spray swath $/ \mathrm{m}$ & 4 \\
\hline No. of Nozzle & 3 & Spray boom length & 2 \\
\hline Flow rate/L $\times$ min $^{-1}$ & $1.5-3.0$ & Flight duration $/ \mathrm{min}$ & 30 \\
\hline Tank capacity/L & 12 & Flight height $/ \mathrm{m}$ & 2 \\
\hline
\end{tabular}

That means by choosing different flight velocities and total nozzle flow rates, the corresponding spraying volume per unit area can be automatically calculated by formula (1). The detail of experimental design is described in the following subsections for Y-shape and CL-shape peach tress, respectively.

\subsection{1. $Y$-shape peach experiments}

Regarding the experimental design for Y-shape peach tree, there are a total of 12 different treatments by choosing various combinations of flight route (intra-row, inter-row), flight velocity $(2,3,4,5 \mathrm{~m} / \mathrm{s})$, total nozzle flow rate $(1.8$, $\left.2.20 \mathrm{~L} \cdot \mathrm{min}^{-1}\right)$, number of spray times $(1,2)$, which are summarized in Table 2 .

In particular, the flight route (intra-row vs inter-row) information for Y-shape peach tree is displayed in Fig 4 along with three sampling points (replicates) for droplet coverage data collection at different peach positions.

\subsection{2. $C L$-shape peach experiments}

The experiment design for CL-shape peach tree is summarized in Table 3 In this case, there are also a total of 12 different treatments by changing flight route (intra-row, inter-row), flight velocity $(2,3,4,5 \mathrm{~m} / \mathrm{s})$ and nozzle flow rate $\left(1.8,2.20,1.60 \mathrm{~L} \cdot \mathrm{min}^{-1}\right)$. In particular, the total nozzle flow rate of $1.60 \mathrm{~L} \cdot \mathrm{min}^{-1}$ with two nozzles is achieved by blocking the middle nozzle of the three nozzles for the case with $2.20 \mathrm{~L} \cdot \mathrm{min}^{-1}$. This is because, as shown in the right picture of Fig 2, there is much spacing (i.e. being soil rather than tree branches) between CL-shape peach rows, spraying by using all three nozzles may waste pesticide by the middle nozzle (i.e. droplets of the middle nozzle are mostly deposited on row space rather than tree leaves). It is noted that the number of spray times is not tested in this case due to logistic constraints. Similarly, the flight route information for CL-shape peach tree is displayed in Fig 5. where the five circles represent the five replicates for droplet data collection. 
Table 2: Different treatment combinations for Y-shape peach tree.

\begin{tabular}{|c|c|c|c|c|c|}
\hline Treatment & Volume/ ha $\cdot L^{-1}$ & Nozzle flow rate $/ L \cdot \min ^{-1}$ & Velocity $\mathrm{m} / \mathrm{s}$ & No. of spray times & Route \\
\hline Y1 & 37.50 & 1.80 & 2 & 1 & Intra \\
\hline $\mathrm{Y} 2$ & 45.00 & 2.20 & 2 & 1 & Intra \\
\hline Y3 & 30.00 & 2.20 & 3 & 1 & Intra \\
\hline Y4 & 22.50 & 2.20 & 4 & 1 & Intra \\
\hline Y5 & 45.00 & 2.20 & 4 & 2 & Intra \\
\hline Y6 & 30.00 & 1.80 & 5 & 2 & Intra \\
\hline Y7 & 37.50 & 1.80 & 2 & 1 & Inter \\
\hline Y8 & 45.00 & 2.20 & 2 & 1 & Inter \\
\hline Y9 & 30.00 & 2.20 & 3 & 1 & Inter \\
\hline Y10 & 22.50 & 2.20 & 4 & 1 & Inter \\
\hline Y11 & 45.00 & 2.20 & 4 & 2 & Inter \\
\hline Y12 & 30.00 & 1.80 & 5 & 2 & Inter \\
\hline
\end{tabular}

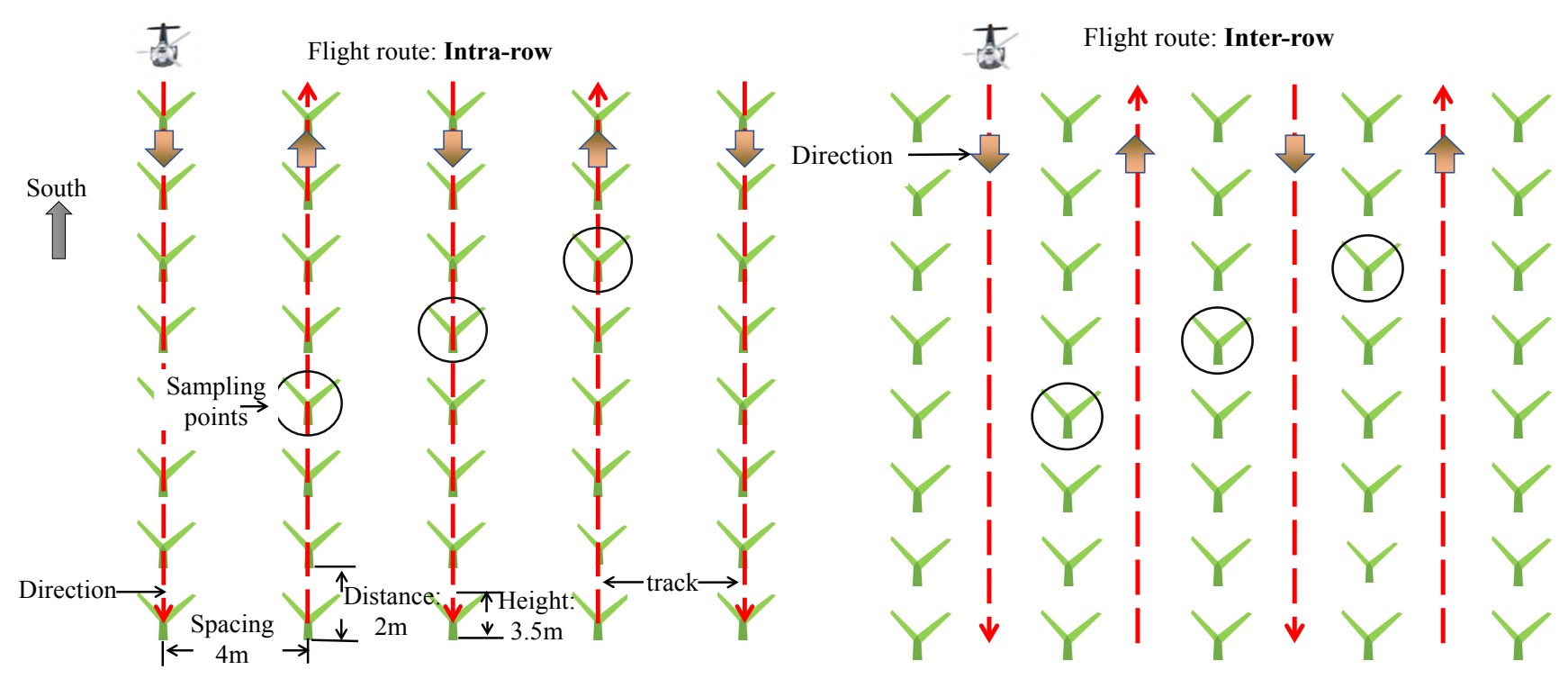

Figure 4: Flight information for Y-shape peach tree: intra-row route (left) and inter-row route (right), where the right dotted line with arrow denotes the flight track with flight direction, the three circles denote the three replicates for droplet data collection. Information about row spacing, tree distance within a row, tree height is also displayed. Note: this image is only for the purpose of illustration, as shown in Fig 2 branches of adjacent rows for Y-shape peach tree are actually overlapped with each other. 
Table 3: Different treatment combinations for CL-shape peach tree.

\begin{tabular}{|c|c|c|c|c|c|}
\hline Treatment & Volume/ ha $\cdot L^{-1}$ & Nozzle flow rate $/ L \cdot \min ^{-1}$ & Velocity $\mathrm{m} / \mathrm{s}$ & No. of Nozzle & Route \\
\hline CL1 & 37.50 & 1.80 & 2 & 3 & Intra \\
\hline CL2 & 25.05 & 1.80 & 3 & 3 & Intra \\
\hline CL3 & 18.75 & 1.80 & 4 & 3 & Intra \\
\hline CL4 & 15.00 & 1.80 & 5 & 3 & Intra \\
\hline CL5 & 45.00 & 2.20 & 2 & 3 & Intra \\
\hline CL6 & 30.00 & 2.20 & 3 & 3 & Intra \\
\hline CL7 & 22.50 & 2.20 & 4 & 3 & Intra \\
\hline CL8 & 18.75 & 2.20 & 5 & 3 & Intra \\
\hline CL9 & 33.00 & 1.60 & 2 & 2 & Inter \\
\hline CL10 & 22.00 & 1.60 & 3 & 2 & Inter \\
\hline CL11 & 16.50 & 1.60 & 4 & 2 & Inter \\
\hline CL12 & 13.35 & 1.60 & 5 & 2 & Inter \\
\hline
\end{tabular}
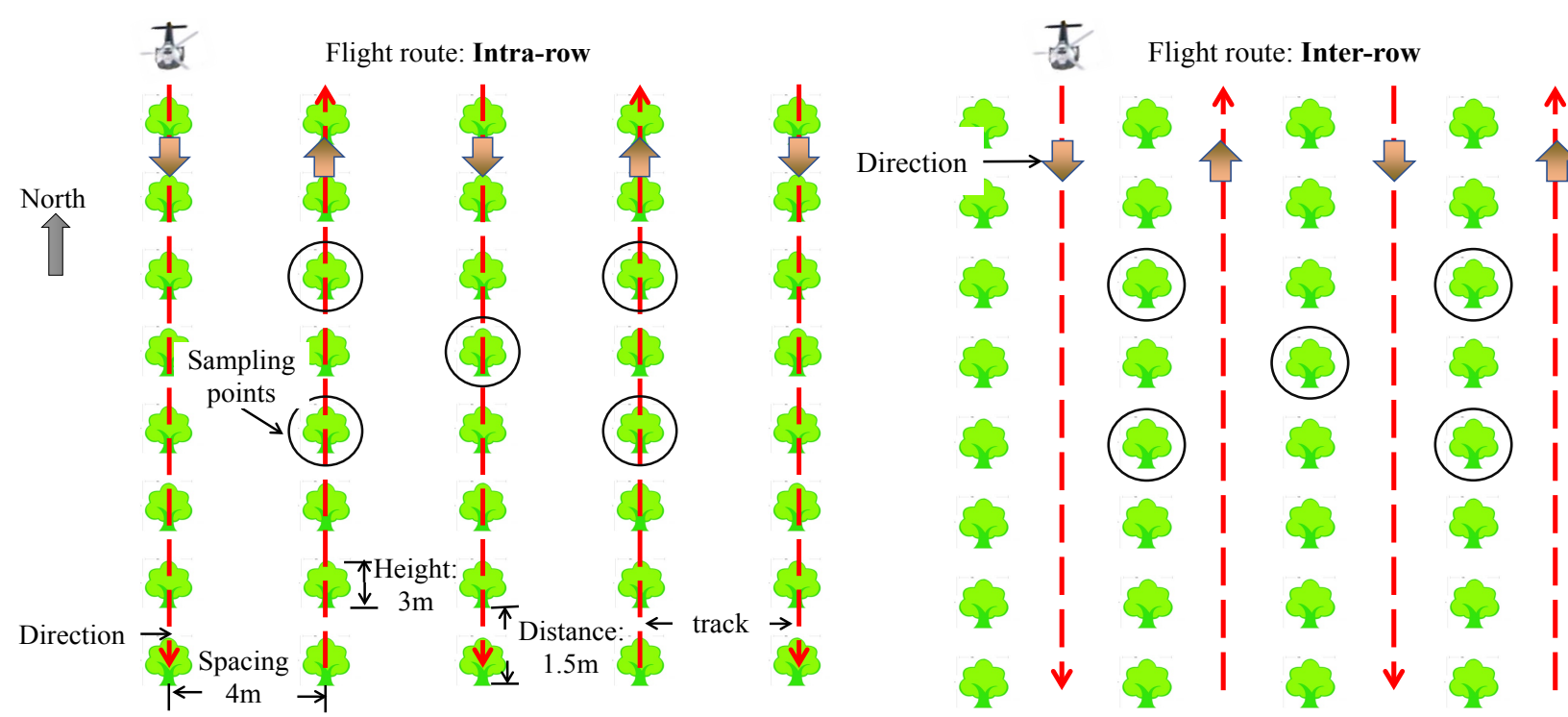

Figure 5: Flight information for CL-shape peach tree: intra-row route (left) and inter-row route (right), where the right dotted line with arrow denotes the flight track with direction, the five circles denote the five replicates for droplet data collection. Information about row spacing, tree distance within a row, tree height is also displayed. 


\subsection{Droplet coverage evaluation}

In this study, droplet coverage rate is selected as the metric to evaluate the spraying effectiveness of various UAV operational parameters [8]. The spraying droplets are first collected by water sensitive papers (WSPs), which are then applied to calculate the droplet coverage rate [26]. In particular, droplet coverage rate is defined by the percentage that WSP is covered by the droplets deposited on it. WSPs $(60 \mathrm{~mm} \times 40 \mathrm{~mm})$ are placed at different positions for both Y-shape and CL-shape peach trees, which are displayed in Fig 6,
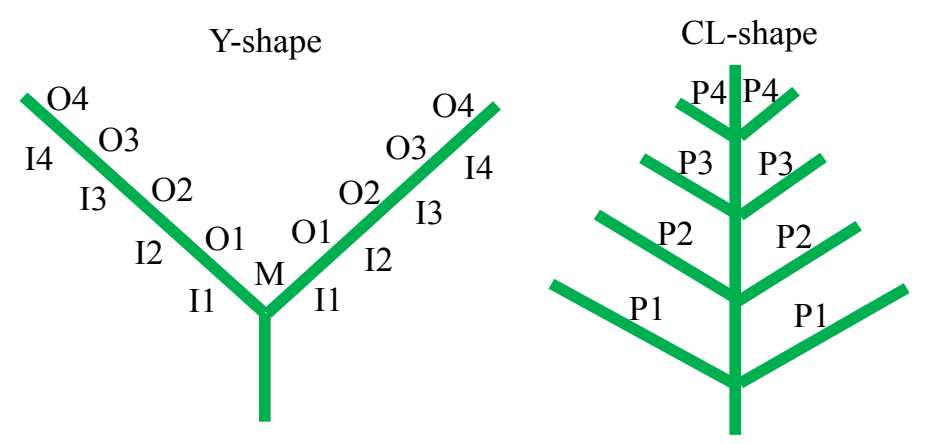

Figure 6: WSP placement positions for both Y-shape and CL-shape peach trees.

As shown in Fig 6, for Y-shape peach tree, WSPs were placed on the two branches symmetrically, where for each branch four WSPs were placed at different positions of the outer (O1, O2, O3, O4) and inner (I1, I2, I3, I4) sides respectively. While for CL-shape peach tree, four layers of branches were selected, and each layer was placed two WSPs symmetrically. The mean value of the droplet coverage rates at two symmetrical positions (except M of Y-shape peach tree) is used to represent the droplet coverage rate at the specific positions (Y-shape) or vertical layers (CL-shape). The WSP cards are collected carefully with tweezers 5-10 minutes after UAV spraying, and immediately placed into a Kraft paper envelope. The WSPs are first scanned by using HP Scanjet G4050 (USA), based on which the droplet coverage rate is analysed by using DepositScan program (USDA, USA) 27.

\subsection{Data analysis}

In this work, lillietest test $[h, p]=\operatorname{lillietest}(x)[28$, where $h$ denotes the decision and $p$ represents $p$-value, is adopted to return a test decision for the null hypothesis that data $x$ comes from a Gaussian distribution, against the alternative that it does not come from a Gaussian distribution. Given the default significance level at $5 \%$, if $p>0.05$ then $h=0$, which means the null hypothesis is kept (i.e. $x$ comes from a Gaussian distribution); and if $p<0.05$ then $h=1$, which means the null hypothesis is rejected (i.e. $x$ does not come from a Gaussian distribution). The rule is 
also summarized in Eq 2 for the ease of readability

$$
x \sim\left\{\begin{array}{ll}
\text { Gaussian distribution } & p \geq 0.05 \& h=0 \\
\text { Non-Gaussian distribution } & p<0.05 \& h=1
\end{array} .\right.
$$

Moreover, the classical t-test [29] with formula $[h, p]=\operatorname{ttest}\left(x, \mu{ }^{\prime}{ }^{\text {Tail }}{ }^{\prime},{ }^{\prime}\right.$ right $\left.^{\prime}\right)$ is adopted to return a test decision for the null hypothesis that data $x$ comes from a Gaussian distribution with mean equal to $\mu$, against the alternative that the mean is greater than $\mu$, where the default significance level is also set at $5 \%$. The rule is summarized in formula 3

$$
\operatorname{mean}(x) \begin{cases}=\mu & p \geq 0.05 \& h=0 \\ >\mu & p<0.05 \& h=1\end{cases}
$$

In addition, the widely used coefficient of variation $(\mathrm{CV})$ is chosen as the metric to assess the uniformity of droplet distribution on tree canopy [30, which is calculated by formula (4)

$$
C V=\frac{S D}{\bar{X}} \times 100 \%, \text { with } \bar{X}=\frac{\sum X_{i}}{n}, S D=\sqrt{\frac{\sum\left(X_{i}-\bar{X}\right)^{2}}{n-1}} .
$$

where $S D$ denotes the standard deviation, $\bar{X}$ represents the average droplet coverage rate; $X_{i}$ is the $i^{t h}$ droplet coverage rate with $n$ being the total number of WSPs in each UAV treatment.

One-way analysis of variance (ANOVA) 31] is also performed for the droplet coverage data at different positions wherever appropriate, where a $p$-value less than 0.05 indicates the existence of significance differences for the means at different positions. If the differences are significant, multiple comparisons are further performed to locate the differences.

\section{Results and discussions}

In this section, the main results are presented, which are divided into two subsections including the results for Y-shape and CL-shape peach trees. Discussions are also provided wherever necessary.

\subsection{Y-shape peach tree}

The results for Y-shape peach tree are first presented, where the average droplet coverage rates under different

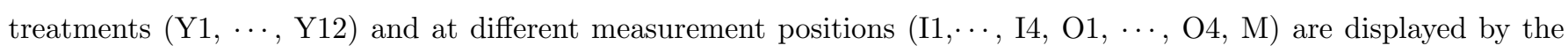
histogram in Fig 7. In addition, mean, standard derivation and CV values in (4) of the droplet coverage rates at different positions are calculated and displayed in Table 4 


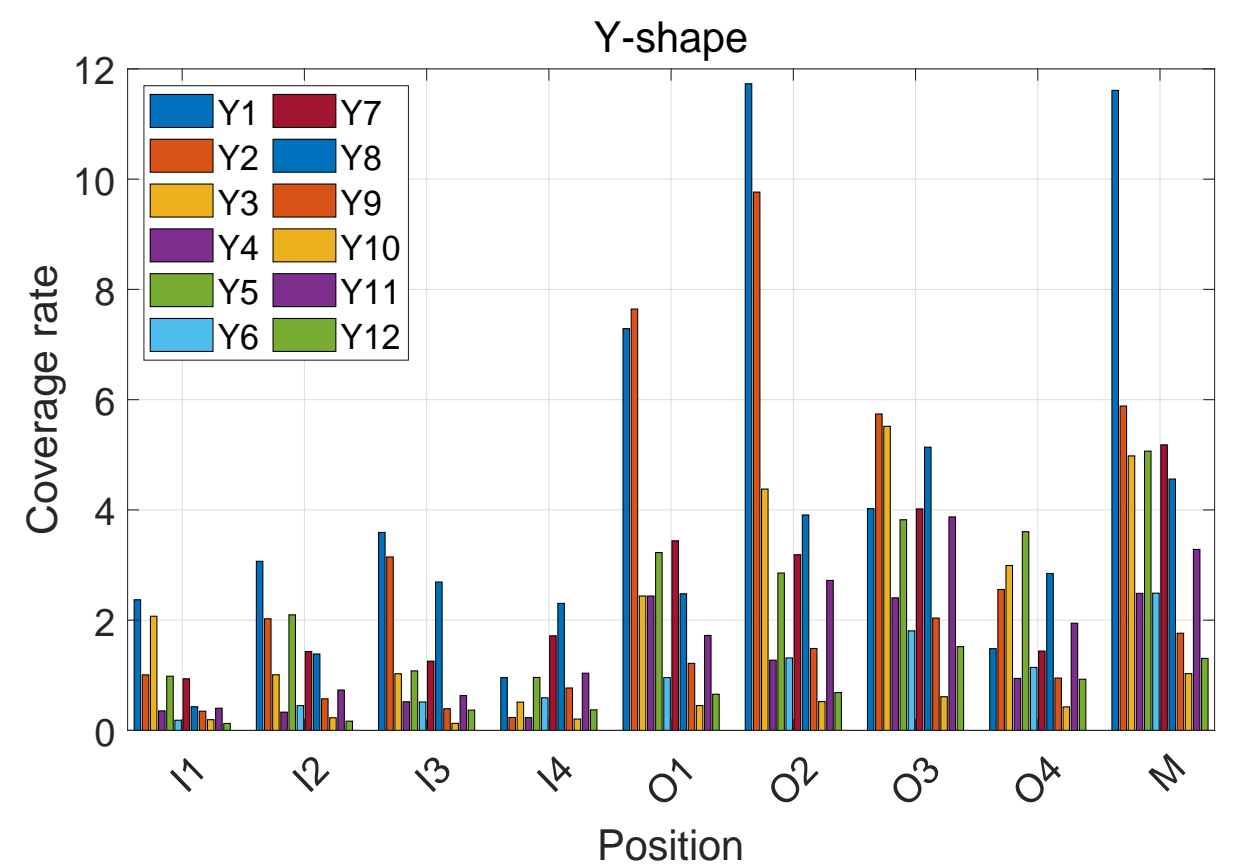

Figure 7: Histogram of the average droplet coverage rates for Y-shape peach tree under different treatments (legend) and at different measurement positions (x-axis).

Table 4: Mean, standard derivation and CV values of the droplet coverage rate at different positions for Y-shape peach.

\begin{tabular}{c|c|c|c|c|c|c|c|c|c}
\hline Metric/Position & I1 & I2 & I3 & I4 & O1 & O2 & O3 & O4 & M \\
\hline Mean & 0.78 & 1.13 & 1.28 & 0.83 & 2.83 & 3.65 & 3.38 & 1.77 & 4.14 \\
\hline Std & 0.74 & 0.90 & 1.19 & 0.64 & 2.37 & 3.56 & 1.67 & 1.01 & 2.88 \\
\hline CV $(\%)$ & 95 & 80 & 93 & 78 & 84 & 97 & 50 & 57 & 70 \\
\hline
\end{tabular}

It follows from Fig 7 and Table 4 that: overall speaking, the average droplet coverage rates at outer positions (O1, O2, O3, O4, M) have significantly higher values than the ones at inner positions (I1, I2, I3, I4). This meets our expectation since outer positions have more chance to deposit droplets than inner ones. This may be one of the limitations of UAV spraying in comparison to manual backpack, which has more freedom in controlling spraying uniformity between outer and inner positions. ANOVA analysis is also performed for the droplet coverage data at inner layer (I1, I2, I3, I4) and outer layer $(\mathrm{O} 1, \mathrm{O} 2, \mathrm{O} 3, \mathrm{O} 4, \mathrm{M})$, respectively, where the $p$-value are 0.47 and 0.18 . The high values (>0.05) imply that there exist no differences among the means for positions at inner or outer layers. This indicates the spraying uniformity by the UAV spraying system in this study for inner layer or outer layer. The effects of UAV operations parameters of interest (e.g. flight route, flight velocity, No. of spray times) on droplet coverage rate are discussed in the following subsections. 


\subsubsection{Effects of flight route}

Different flight routes (intra-row, inter row) may have different effects on droplet coverage rate, which is tested in this part for Y-shape peach tree. In particular, treatments Y1-Y6 are under intra-row route, and Y7-Y12 are under inter-row route. The operational parameters (e.g. spraying volume per unit, total nozzle flow rate, flight velocity, number of spray times) are kept the same for the treatment pairs described in the legend of Fig 8 , where the only variable of concern is flight route. The error histograms (Y1-Y7, Y2-Y8, Y3-Y9, Y4-Y10, Y5-Y11, Y6-Y12) for the average droplet coverage rate are plotted in the left plot of Fig 8 , and the corresponding box plots at different measurement positions are displayed in the right plot of Fig 8

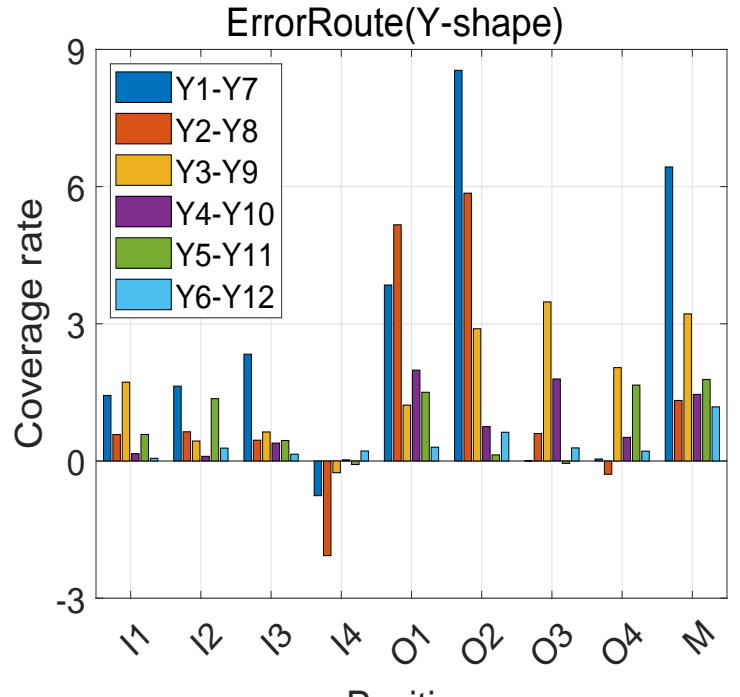

Position

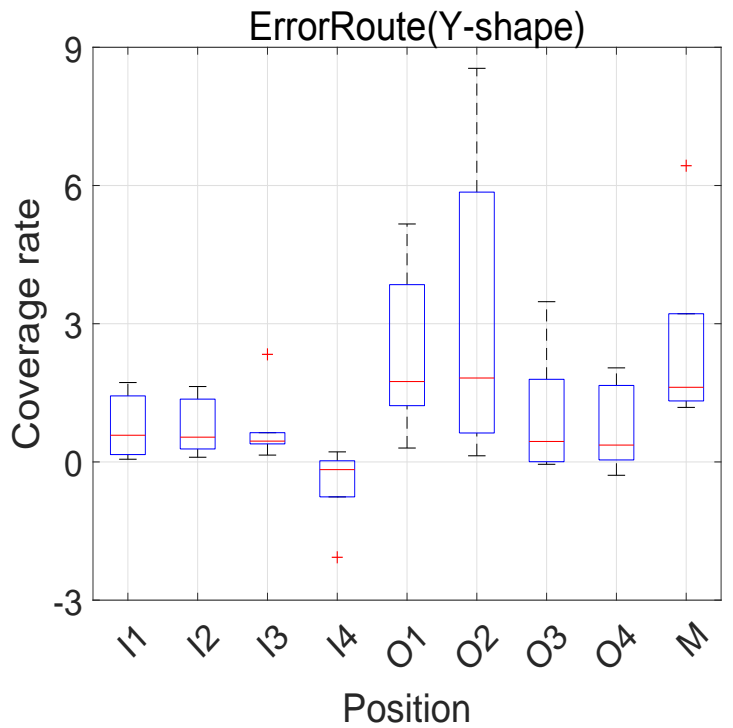

Position

Figure 8: Histogram of error droplet coverage rates between different treatment pairs (left); box plot of the error droplet coverage rates at different positions, assessing the effects of flight route.

In addition, lillietest test in Eq (2) and t-test in (3) are also applied to the error droplet coverage rate data at different positions to test whether or not the data comes from a Gaussian distribution and whether or not the mean value is significantly larger than zero (i.e. whether the droplet coverage rate of intra-row route is significantly larger than that of inter-row route or not). The test results are summarized in Table 5

Table 5: Lillietest and t-test results for the error droplet coverage data at different positions.

\begin{tabular}{c|c|c|c|c|c|c|c|c|c}
\hline Test/Position & I1 & I2 & I3 & I4 & O1 & O2 & O3 & O4 & M \\
\hline Lillietest (p-value): Gaussian vs Non-Gaussian & 0.2 & 0.39 & 0.41 & 0.18 & 0.33 & 0.25 & 0.13 & 0.33 & 0.06 \\
\hline t-test (p-value): mean =0 vs mean $>0$ & 0.02 & 0.02 & 0.04 & 0.9 & 0.01 & 0.04 & 0.07 & 0.06 & 0.01 \\
\hline
\end{tabular}

It follows from Fig 8 that: intra-row route has higher droplet coverage rates than inter-row route at all positions 
except I4 (i.e. the errors in left plot are larger than zero, and the mean errors represented by the red bars in right plot are larger than zero), although the margins vary at different positions and under different UAV operational parameters. In addition, it can be quantitatively drawn from Table 5 that: (1) the error droplet coverage rates between intra-row route and inter-row rate follow a Gaussian distribution (via lillietest); (2) the droplet coverage rate of intra-row route is significantly larger than that of inter-row route at I1, I2, I3, O1, O2 and M with a significance level of 5\% (via t-test). In particular, the average droplet margins at positions $\mathrm{O} 1, \mathrm{O} 2$ and $\mathrm{M}$ are actually over 1.5. Overall speaking, it can be concluded that intra-row route is significantly more effective than inter-row route at most peach tree positions for Y-shape peach tree. Therefore, in practical applications, intra-row route is recommended for Y-shape peach tree.

\subsubsection{Effects of flight velocity}

Given the total nozzle flow rate $\left(2.20 \mathrm{~L} \cdot \mathrm{min}^{-1}\right)$, the effects of flight velocity on droplet coverage rate are further tested for both intra-row and inter-row routes. In particular, for intra-row route Y2 $(2 \mathrm{~m} / \mathrm{s}), \mathrm{Y} 3(3 \mathrm{~m} / \mathrm{s})$ and $\mathrm{Y} 4(4 \mathrm{~m} / \mathrm{s})$ are compared; while for inter-row route Y8 $(2 \mathrm{~m} / \mathrm{s}), \mathrm{Y} 9(3 \mathrm{~m} / \mathrm{s})$ and $\mathrm{Y} 10(4 \mathrm{~m} / \mathrm{s})$ are compared. The average droplet coverage rates for various treatments under different routes are displayed by the histograms in Fig 9 .
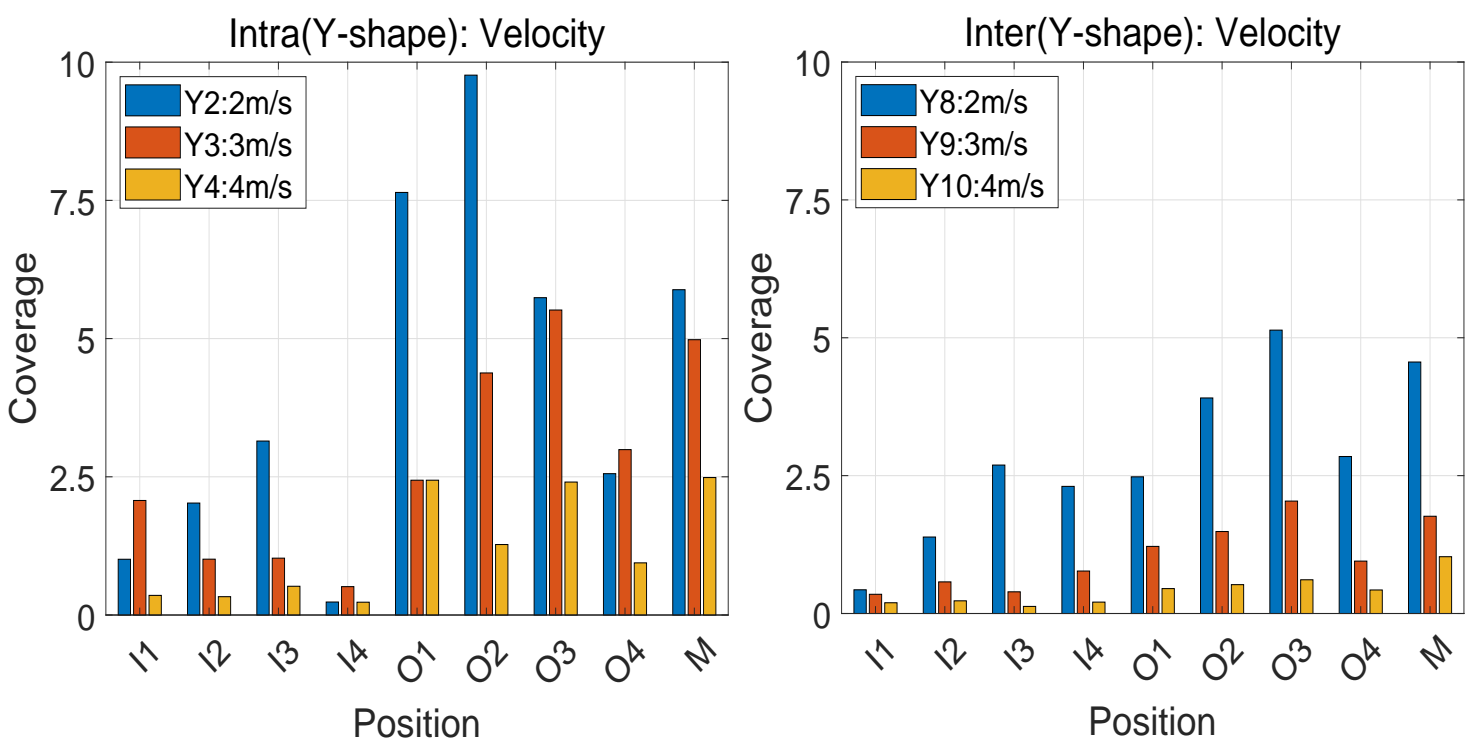

Figure 9: Histogram of droplet coverage rates for Y2/Y3/Y4 (intra-row) and Y8/Y9/Y10 (inter-row), assessing the effects of flight velocity.

In addition, mean, standard derivation and CV values in (4) of the droplet coverage rate at inner layer (I1, I2, I3, I4) and outer layer (O1, O2, O3, O4 and M) (note: different positions of inner and outer layers are seen as different measurements for the particular layer) are calculated and displayed in Table 6. On this basis, a linear regression analysis between the coverage rate and flight velocity for different Route/Layer combinations is also performed, where 
the correlation coefficient $(\mathrm{CC})$ and $p$-value $(p<0.05$ means significant) testing the significance of the linear regression model are shown in the rightmost columns of Table 6 .

Table 6: Mean, standard derivation and CV values of the droplet coverage rates on peach canopy under different velocities and different route/layer combinations, and linear correlation analysis with $\mathrm{CC}$ and p-value.

\begin{tabular}{c|c|c|c|c|c}
\hline Route/velocity & $2 \mathrm{~m} / \mathrm{s}$ & $3 \mathrm{~m} / \mathrm{s}$ & $4 \mathrm{~m} / \mathrm{s}$ & $\mathrm{CC}$ & $\mathrm{p}$-value \\
\hline Intra-row: Inner & $1.60 \pm 1.26: 79 \%$ & $1.16 \pm 0.65: 57 \%$ & $0.36 \pm 0.12: 33 \%$ & -0.58 & 0.049 \\
\hline Intra-row: Outer & $6.32 \pm 2.66: 42 \%$ & $4.06 \pm 1.31: 32 \%$ & $1.91 \pm 0.74: 39 \%$ & -0.75 & 0.001 \\
\hline Inter-row: Inner & $1.70 \pm 1.01: 59 \%$ & $0.52 \pm 0.19: 37 \%$ & $0.19 \pm 0.04: 23 \%$ & -0.75 & 0.005 \\
\hline Inter-row: Outer & $3.79 \pm 1.12: 30 \%$ & $1.49 \pm 0.43: 29 \%$ & $0.61 \pm 0.25: 40 \%$ & -0.88 & 0 \\
\hline
\end{tabular}

It follows from Fig 9 that: (1) for intra-row route, the droplet coverage rate generally decreases with the increase of flight velocity although there are some exceptions at position I1, I4 and O4 (note: the likely reason is the effects of measurement errors); (2) for inter-row route, the droplet coverage rate strictly decreases with the increase of flight velocity at all positions. These conclusions can also be quantitatively drawn from Table 6 , since the correlation coefficients are all negative. In addition, inter-row route results in a more uniform spraying performance (i.e. with a smaller value of CV). Moreover, the linear relationship between coverage rate and flight velocity for different route/layer combinations is all significant (i.e. $p$-values are all less than 0.05). Overall speaking, the increase of flight velocity will significantly decrease the droplet coverage rate, which actually meets our expectation and is also consistent with previous results [13, 14].

\subsubsection{Effects of No. of spray times}

Given the total nozzle flow rate and the spray volume per unit area, one can spray twice if the flight velocity is doubled. In this part, we investigate whether doubling the number of spray times can improve spraying effectiveness or not. In particular, for intra-row route, Y2 (once) and Y5 (twice) are compared; while for inter-row route, Y8 (once) and Y11 (twice) are compared. The error histogram (Y2-Y5, Y8-Y11) for the average droplet coverage rate is plotted in Fig 10. In addition, mean, standard derivation and CV values of the error droplet coverage rates on peach canopy are also calculated, where the results for intra-row and inter-row routes are 1.36 $\pm 2.54: 187 \%$ and $0.82 \pm 0.76$ : $93 \%$, respectively. It is noted that the data for intra-row route does not satisfy a Gaussian distribution by Lillietest test and therefore t-test is not performed to see whether the mean value is significantly higher than zero.

It follows from Fig 10 and the above statistics that: (1) for intra-row route, "spraying once" has a higher droplet 


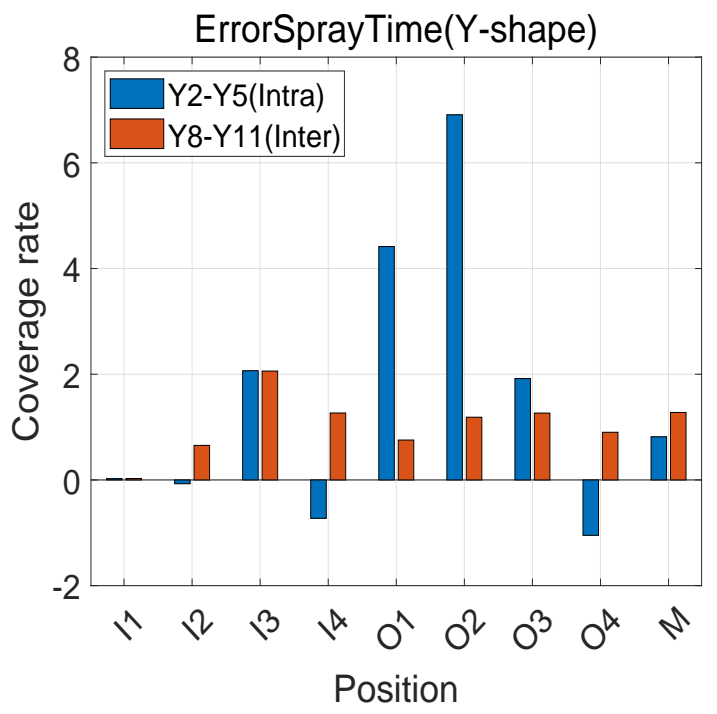

Figure 10: Histogram of error coverage rates for Y2/Y5 (intra-row) and Y8/Y11 (inter-row), assessing the effects of No. of spray times.

coverage value than "spraying twice" at position I3, O1, O2, O3 and M, has a similar value at I1 and I2, and has a smaller value at I4 and O4; (2) for inter-row route, "spraying once" has a higher droplet coverage value over "spraying twice" at all tested positions. Therefore, overall speaking, it can be concluded that given the spraying volume per unit doubling the number of spray times decreased the performance of both intra-row and inter-row routes for Y-shape peach tree. The likely reason for performance degradation is that: by doubling the flight velocity, the downwash airflow generated by the UAV rotors may become weak, increasing the chance of droplet drift. Therefore, "spraying once" is recommended in practical applications for peach tree of Y-shape.

\subsection{CL-shape peach tree}

In this part, the results for CL-shape peach tree are presented, where the average droplet coverage rates under different treatments $(\mathrm{CL} 1, \cdots, \mathrm{CL12})$ and at different positions $(\mathrm{P} 1, \cdots, \mathrm{P} 4)$ are displayed by the histograms in Fig 11. In addition, mean, standard derivation and CV values in (4) of the droplet coverage rate at different positions are calculated and displayed in Table 7 .

Table 7: Mean, standard derivation and CV values of the droplet coverage rate at different positions for CL-shape peach.
\begin{tabular}{c|c|c|c|c}
\hline Metric/Position & P1 & P2 & P3 & P4 \\
\hline Mean \pm Std: CV & $0.81 \pm 0.58: 71 \%$ & $0.87 \pm 0.38: 43 \%$ & $0.80 \pm 0.39: 49 \%$ & $1.46 \pm 0.86: 59 \%$
\end{tabular}

It follows from Fig 11 and Table 7 that: overall speaking, the average droplet coverage rates at positions P1, P2, and P3 have similar values $(0.81,0.87,0.80)$, while position P4 (top layer) has a higher value (1.46). Moreover, ANOVA 


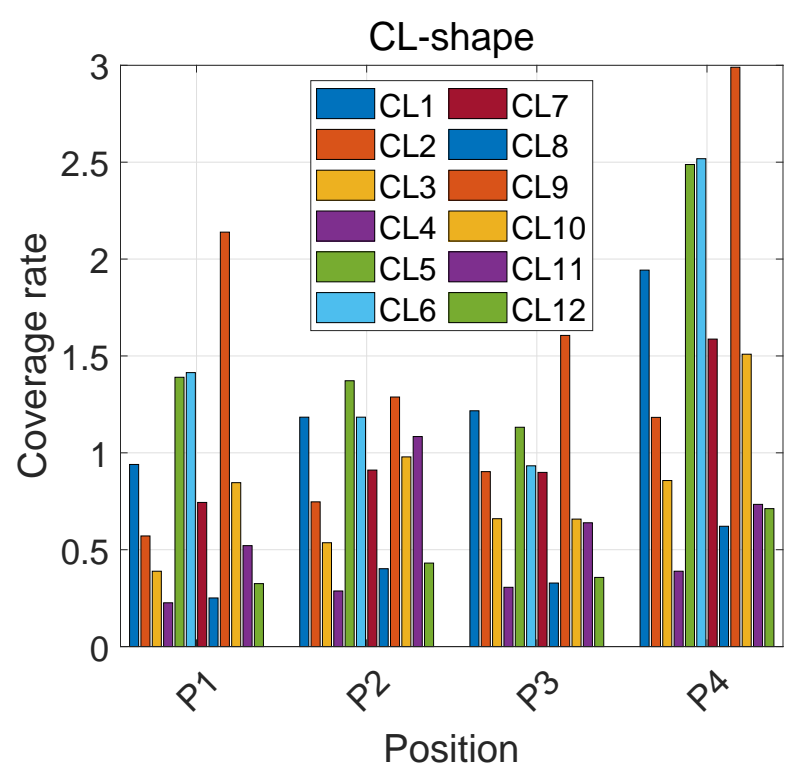

Figure 11: Histogram of the average droplet coverage rates for CL-shape peach tree under different treatments (legend) and at different measurement positions (x-axis).

analysis is also performed, where the $p$-value for all four groups is 0.022 , indicating that differences among the means of different positions are significant. While the $p$-value of ANOVA for the lower three layers is 0.93 , indicating the uniformity at lower three layers. Then multiple comparisons further show that $P 1$ and $P 3$ are significantly different from P4. Although there is a high droplet coverage at top layer P4, the droplet coverages at P1, P2 and P3 also demonstrate the increased penetrability by downward airflow generated by UAV rotors. The effects of UAV operations parameters of interest (e.g. flight route, flight velocity and total nozzle flow rate) on droplet coverage rate are discussed in the following subsections.

\subsubsection{Effects of flight route}

The effects of flight route are first tested for CL-shape peach tree. In particular, treatments CL1-CL4 (Group 1) are under intra-row route while with a total nozzle flow rate of $1.8 \mathrm{~L} \cdot \mathrm{min}^{-1}$, treatment CL5-CL8 (Group 2) are under intra-row route while with a total nozzle flow rate of $2.2 \mathrm{~L} \cdot \mathrm{min}^{-1}$, and treatments CL9-CL12 (Group 3) are under inter-row rate while with a total nozzle flow rate of $1.6 \mathrm{~L} \cdot \mathrm{min}^{-1}$. The comparisons are made between Group 1 and Group 3, and Group 2 and Group 3, where the error droplet coverage rates for the treatment pairs with the same flight velocity are computed. Their error histograms (Group 1 vs Group 3: CL1-CL9, CL2-CL10, CL3-CL11, CL4-CL12; Group 2 vs Group 3: CL5-CL9, CL6-CL10, CL7-CL11, CL8-CL12) and the corresponding box plots at different measurement positions are displayed in Fig 12 and Fig 13 . It is noted that different from Section 3.1 .1 
statistical significance (i.e. the test that the mean error is significantly larger than zero or not) is not performed due to the small number of samples at different positions.
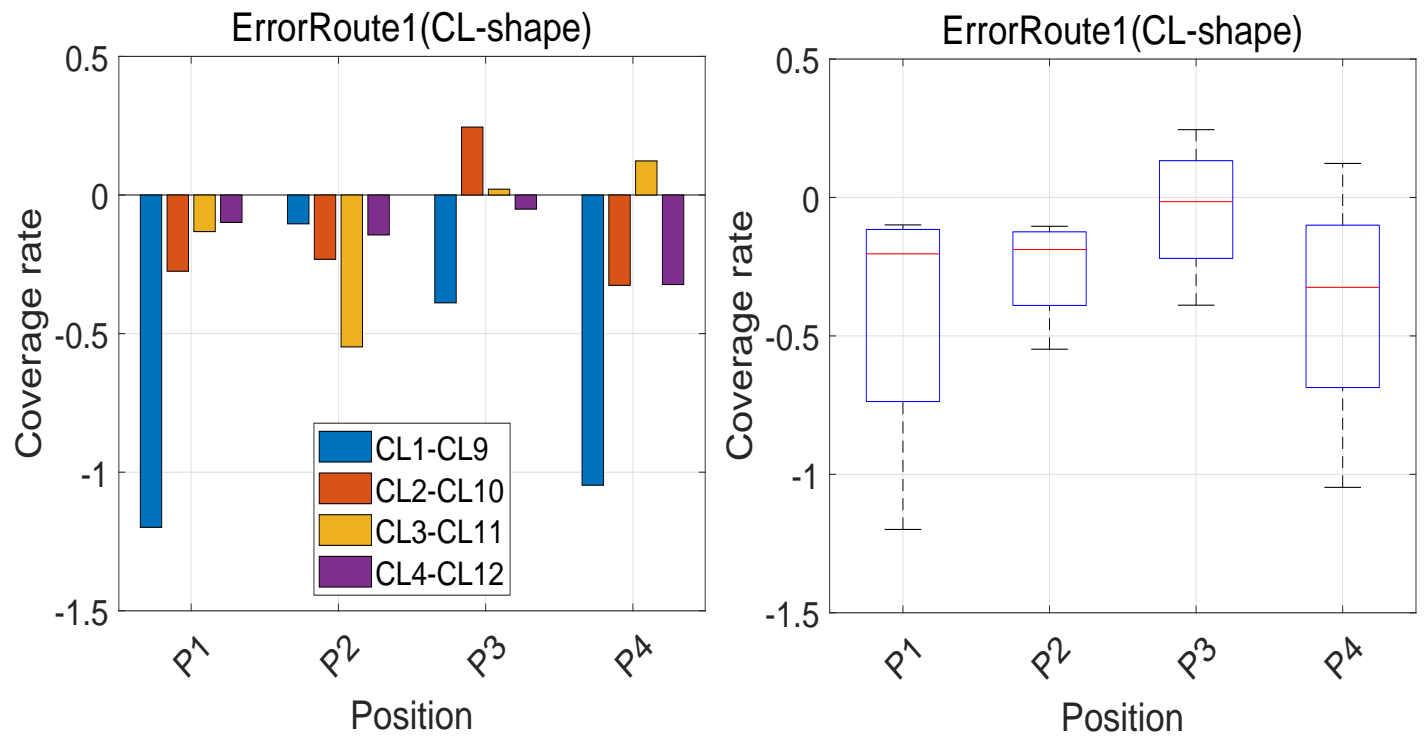

Figure 12: Histogram and box plot of error droplet coverage rate, assessing the effects of flight route.
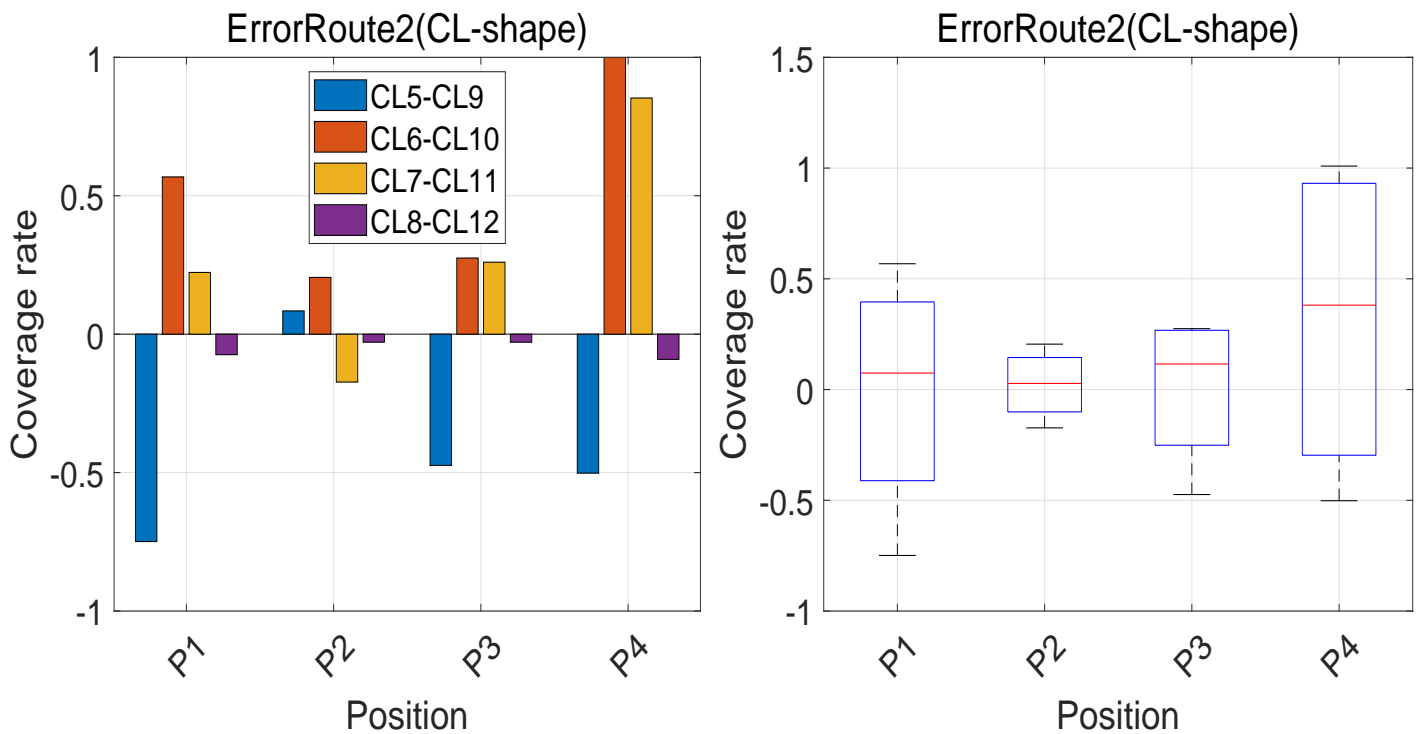

Figure 13: Histogram and box plot of error droplet coverage rate, assessing the effects of flight route.

It follows from Fig 12 and Fig 13 that: (1) for Group 1 (intra-row route with a total nozzle flow rate of 1.80) vs Group 3 (inter-row route with a total nozzle flow rate of 1.60), inter-row route strategy not only saves a nozzle flow rate of $0.2 \mathrm{~L} \cdot \mathrm{min}^{-1}$, but also increases the droplet coverage rate at positions $\mathrm{P} 1, \mathrm{P} 2$, and $\mathrm{P} 4$ while with a similar droplet coverage rate at P3; (2) for Group 2 (intra-row route with a total nozzle flow rate of 2.20) vs Group 3 (inter-row route with a total nozzle flow rate of 1.60), inter-row route strategy has a slightly lower droplet coverage 
rate (less than 0.2 at positions $\mathrm{P} 1, \mathrm{P} 2$, and $\mathrm{P} 3$; and about 0.4 at position $\mathrm{P} 4$ ) but only takes about $73 \%$ of the nozzle flow rate. Therefore, overall speaking, it is concluded that inter-row route obtains better spraying performance than intra-row route for CL-shape peach tree in terms of droplet coverage rate and pesticide saving. As a result, inter-row route is recommended for CL-shape tree in practical applications.

\subsubsection{Effects of flight velocity}

The effects of flight velocity on droplet coverage rate are further tested under different flight routes and total nozzle flow rates. In particular, for intra-row route, two groups are compared including the ones with a total nozzle flow rate of $1.80 \mathrm{~L} \cdot \mathrm{min}^{-1}: \operatorname{CL} 1(2 \mathrm{~m} / \mathrm{s})$, CL2 $(3 \mathrm{~m} / \mathrm{s})$, CL3 $(4 \mathrm{~m} / \mathrm{s})$, CL4 $(5 \mathrm{~m} / \mathrm{s})$, and the ones with a total nozzle flow rate of $2.20 \mathrm{~L} \cdot \mathrm{min}^{-1}$ : CL5 $(2 \mathrm{~m} / \mathrm{s})$, CL6 $(3 \mathrm{~m} / \mathrm{s})$, CL7 $(4 \mathrm{~m} / \mathrm{s})$, CL8 (5m/s); while for inter-row route CL9 (2m/s), CL10 $(3 \mathrm{~m} / \mathrm{s})$, CL11 $(4 \mathrm{~m} / \mathrm{s})$, CL12 $(5 \mathrm{~m} / \mathrm{s})$ are compared. The average droplet coverage rates under various conditions are displayed by the histograms in Fig 14
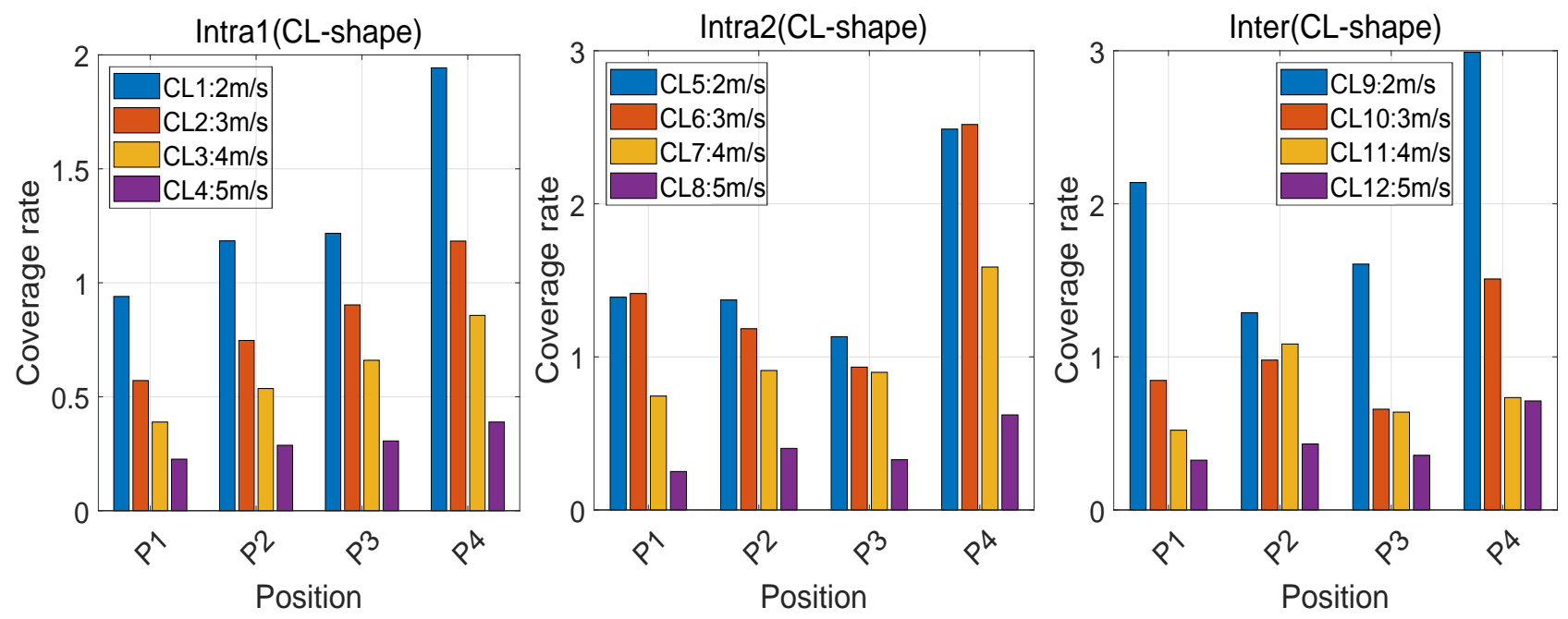

Figure 14: Histogram of droplet coverage rates for CL1/CL2/CL3/CL4 (intra-row with a total nozzle flow rate of 1.80), CL5/CL6/CL7/CL8 (intra-row with a total nozzle flow rate of 2.20) and CL9/CL10/CL11/CL12 (inter-row), assessing the effects of flight velocity.

In addition, mean, standard derivation and $\mathrm{CV}$ values of the droplet coverage rates on peach canopy (different positions are seen as different measurements for the peach canopy) are calculated and displayed in Table 8 . On this basis, a linear regression analysis between the coverage rate and flight velocity for different Route/nozzle flow rate combinations is also performed, where the $\mathrm{CC}$ and $p$-value are shown in the rightmost columns of the table.

It follows from Fig 14 that: under different conditions (e.g. flight route, total nozzle flow rate), the effects of flight velocity on droplet coverage rate are very similar, i.e., the increase of flight velocity decreases the droplet coverage 
Table 8: Mean, Std and CV values of the droplet coverage rate on peach canopy, and linear correlation analysis with CC and p-value.

\begin{tabular}{c|c|c|c|c|c|c}
\hline Route/velocity & $2 \mathrm{~m} / \mathrm{s}$ & $3 \mathrm{~m} / \mathrm{s}$ & $4 \mathrm{~m} / \mathrm{s}$ & $5 \mathrm{~m} / \mathrm{s}$ & $\mathrm{CC}$ & $\mathrm{p}-\mathrm{value}$ \\
\hline $\operatorname{Intra}(1.8)$ & $1.32 \pm 0.43: 33 \%$ & $0.85 \pm 0.26: 31 \%$ & $0.61 \pm 0.20: 32 \%$ & $0.30 \pm 0.07: 22 \%$ & -0.84 & $1 \times 10^{-4}$ \\
\hline $\operatorname{Intra}(2.2)$ & $1.60 \pm 0.61: 38 \%$ & $1.51 \pm 0.70: 46 \%$ & $1.04 \pm 0.38: 36 \%$ & $0.40 \pm 0.16: 40 \%$ & -0.70 & $2.4 \times 10^{-3}$ \\
\hline $\operatorname{Intra}(1.6)$ & $2.01 \pm 0.74: 37 \%$ & $1.00 \pm 0.37: 37 \%$ & $0.74 \pm 0.24: 33 \%$ & $0.47 \pm 0.18: 39 \%$ & -0.79 & $3 \times 10^{-4}$ \\
\hline
\end{tabular}

rate. This observation can also be quantitatively confirmed by the results in Table 8 by observing that the CC values are all negative. In addition, the linear relationship between droplet coverage rate and flight velocity is also significant for all cases, since the $p$-values are all less than 0.05. This conclusion is consistent with the Y-shape result in Section 3.1 .2 and also the previous results 13,14

\subsubsection{Effects of total nozzle flow rate}

The effects of total nozzle flow rate on droplet coverage rate are then tested for CL-shape peach tree. In particular, treatments CL1-CL4 (Group 1) with a total nozzle flow rate of $1.8 \mathrm{~L} \cdot \mathrm{min}^{-1}$ and treatments CL5-CL8 (Group 2) with a total nozzle flow rate of $2.2 \mathrm{~L} \cdot \mathrm{min}^{-1}$ are compared. For the treatments with the same flight velocity in Group 1 and Group 2, histograms (CL5-CL1, CL6-CL2, CL7-CL3, CL8-CL4) and box plots of their error coverage rates are displayed in Fig 15. In addition, lillietest test and t-test are also applied to the error data at different positions,
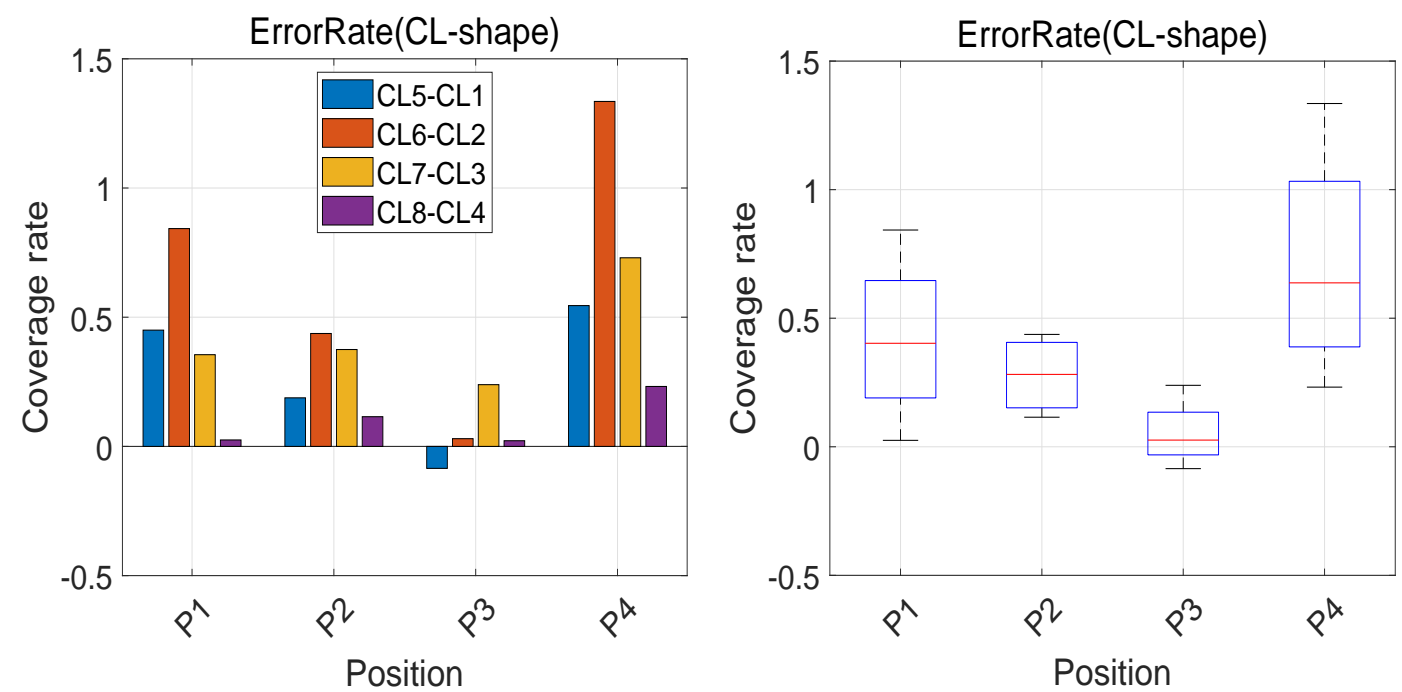

Figure 15: Histogram and box plot of error droplet coverage rates, assessing the effects of total nozzle flow rate.

where the results are summarized in Table 9 ,

It follows from Fig 15 that for intra-row route increasing the total nozzle flow rate from 1.8 to $2.2 \mathrm{~L} \cdot \mathrm{min}^{-1}$ can increase the droplet coverage rate at different layers of CL-shape peach tree, where the top layer P4 has the most 
Table 9: Lillietest and t-test results for the error droplet coverage data for CL-shape at different positions.

\begin{tabular}{c|c|c|c|c}
\hline Test & P1 & P2 & P3 & P4 \\
\hline Lillietest (p-value): Gaussian vs Non-Gaussian & 0.50 & 0.50 & 0.17 & 0.50 \\
\hline t-test (p-value): mean $=0$ vs mean $>0$ & 0.04 & 0.02 & 0.25 & 0.03 \\
\hline
\end{tabular}

significant increase followed by P1, P2 and P3. In addition, it can also be quantitatively seen from Table 9 that: (1) that error droplet coverage rate data at different positions satisfy a Gaussian distribution; (2) increasing the nozzle flow rate can significantly improve the droplet coverage rate at positions P1, P2 and P4, where the improvement at P3 is insignificant at the significance level of 0.05. Considering that inter-row route is recommended for CL-shape peach tree in Section 3.2.1, the effects of nozzle flow rate should also be assessed for inter-row route in future work. More importantly, the quantitative relationship should be established.

\subsection{Further comparisons}

In this part, further comparisons are made, which include the comparison between this work and existing studies on orchard trees, and the comparison between Y-shape and CL-shape peach trees in droplet distribution. The comparisons are summarized as below.

Some of the results in this study are consistent with some existing work on orchard trees (citrus in particular) [20, 21, 22]. For example, tree shape is shown to have significant effects on droplet coverage [22]; the droplet coverage rate is negatively correlated with flight velocity [20]. However, this study is also significantly different from the preceding studies. First, the shape of peach tree (Y-shape or CL-shape) is significantly different from citrus tree (triangle or inverted-triangle shape by manual pruning). Secondly, multi-rotor UAV is used in previous studies for citrus orchard, while gas powered helicopter is used for peach orchard in this study. Different types of UAVs usually result in distinct downwash air flow distribution, significantly affecting droplet distribution [25]. Thirdly, different parameters affecting droplet distribution are considered in this study, for example, flight route and number of spray times are newly investigated in this study.

The comparison of operational parameters between Y-shape and CL-shape peach trees is summarized in Table 10 for the ease of readability. In particular, "NA" means that experiments are not available for that case due to logistic constraints. The detailed explanations about the comparisons between Y-shape and CL-shape peach trees are referred to Section 4 
Table 10: Comparison between Y-shape and CL-shape peach trees.

\begin{tabular}{c|c|c|c|c|c}
\hline Tree shape & Uniformity & Flight route & Flight velocity & Spray time & Nozzle flow rate \\
\hline Y-shape & Inner \& Outer (Yes) & Intra-row & Negative, significant & Once & NA \\
\hline CL-shape & Top > Lower three & Inter-row & Negative, significant & NA & Significant (some) \\
\hline
\end{tabular}

\section{Conclusions and future work}

Small-scale plant protection UAVs are emerging spraying tools for orchard trees due to their high efficiency, effectiveness and flexibility. In order to realize their potentials, this study aimed to investigate the effects of their operational parameters on droplet distribution (at different positions/layers) for peach trees of various shapes including Y-shape and CL-shape. Field test experiments were performed in Shandong Province, China by using gas powered helicopter 3WQF120-12, where water sensitive papers were used to calculate the droplet coverage rate at different positions, evaluating the spraying performance. The parameters of interest in this study included flight route (intrarow, inter-row), flight velocity (four levels: $2,3,4,5 \mathrm{~m} / \mathrm{s}$ ), number of spray times (1 vs 2 ) and nozzle flow rate (1.8 vs $\left.2.2 \mathrm{~L} \cdot \mathrm{min}^{-1}\right)$. The following results are obtained by the experiments:

(1) The spraying uniformity was different between Y-shape and CL-shape. For Y-shape peach, the droplet coverage at outer layer was significantly higher than lower layers, however, the droplet coverage rate was uniform for positions at inner or outer layers. For CL-shape peach the droplet coverage at top layer was significantly higher than lower three layers, however, the lower three layers shared a similar droplet coverage rate.

(2) The suitable flight route was different, which was Intra-row route for Y-shape and Inter-row route for CL-shape.

(3) The effect of flight velocity on droplet coverage rate was similar for both tree shapes, where a significantly negative correlation was observed.

(4) For Y-shape peach, given spray volume per unit area "spraying once" was more preferable than "spraying twice".

(5) For CL-shape peach, increasing the total nozzle flow rate from 1.8 to $2.2 \mathrm{~L} \cdot \mathrm{min}^{-1}$ can significantly improve the droplet coverage at specific positions including top layer and bottom two layers .

This paper makes a good progress in optimising flight and other parameters in order to maximise the spray performance and reduce the use of pesticide with respect to different tree shapes and other conditions. However, there is still much room for further development in order that the developed systems can be applied in real-life application for orchard spraying. The following aspects are identified 
(i) The quantitative relationship/model between droplet coverage rate and flight parameters (e.g. flight velocity, nozzle flow rate) is yet to be quantified for peach trees.

(ii) The number of spray times for CL-shape peach should also be investigated. More importantly, the underlying reasons about the effects of No. spray times on droplet coverage should be investigated by measuring the degree of downwash airflow by the UAV rotors.

(iii) The actual performance for the control of pests and diseases should also be evaluated for peach trees against conventional manual backpack approach before the optimized operational strategy being commercialized.

\section{Acknowledgements}

Yanhua Meng, Jianli Song and Yubin Lan are supported by National Key Research and Development Program of China (2017YFE0122400), the National Key Research and Development Plan Program of China (2016YFD0200700), the Leading Talents of Guangdong Province Program (2016LJ06G689), Science and Technology Planning Project of Guangdong Province (2017B010117010), The 111 Project (D18019). Jinya Su and Wen-Hua Chen are supported by the UK-China Agritech Challenge-Utilizing Earth Observation and UAV Technologies to Deliver Pest and Disease Products and Services to End Users in China (BBSRC with Grant number BB/S020977/1).

\section{References}

[1] H. C. J. Godfray, J. R. Beddington, I. R. Crute, L. Haddad, D. Lawrence, J. F. Muir, J. Pretty, S. Robinson, S. M. Thomas, and C. Toulmin, "Food security: the challenge of feeding 9 billion people," science, vol. 327, no. 5967, pp. 812-818, 2010.

[2] S. Guo, J. Li, W. Yao, Y. Zhan, Y. Li, and Y. Shi, "Distribution characteristics on droplet deposition of wind field vortex formed by multi-rotor uav," PloS one, vol. 14, no. 7, p. e0220024, 2019.

[3] J. Su, C. Liu, M. Coombes, X. Hu, C. Wang, X. Xu, Q. Li, L. Guo, and W.-H. Chen, "Wheat yellow rust monitoring by learning from multispectral uav aerial imagery," Computers and electronics in agriculture, vol. 155, pp. 157-166, 2018.

[4] J. Su, C. Liu, X. Hu, X. Xu, L. Guo, and W.-H. Chen, "Spatio-temporal monitoring of wheat yellow rust using uav multispectral imagery," Computers and electronics in agriculture, vol. 167, p. 105035, 2019.

[5] Y. Lan and S. Chen, "Current status and trends of plant protection uav and its spraying technology in china," International Journal of Precision Agricultural Aviation, vol. 1, no. 1, 2018. 
[6] W.-C. Qin, B.-J. Qiu, X.-Y. Xue, C. Chen, Z.-F. Xu, and Q.-Q. Zhou, "Droplet deposition and control effect of insecticides sprayed with an unmanned aerial vehicle against plant hoppers," Crop Protection, vol. 85, pp. 79-88, 2016.

[7] J. Li, Y. Shi, Y. Lan, and S. Guo, "Vertical distribution and vortex structure of rotor wind field under the influence of rice canopy," Computers and Electronics in Agriculture, vol. 159, pp. 140-146, 2019.

[8] Y. Meng, J. Song, Y. Lan, G. Mei, Z. Liang, and Y. Han, "Harvest aids efficacy applied by unmanned aerial vehicles on cotton crop," Industrial Crops and Products, vol. 140, p. 111645, 2019.

[9] B. K. Fritz, I. W. Kirk, W. C. Hoffmann, D. E. Martin, V. L. Hofman, C. Hollingsworth, M. McMullen, and S. Halley, "Aerial application methods for increasing spray deposition on wheat heads," Applied Engineering in Agriculture, vol. 22, no. 3, pp. 357-364, 2006.

[10] R. Derksen, H. Zhu, H. Ozkan, R. Hammond, A. Dorrance, and A. Spongberg, "Determining the influence of spray quality, nozzle type, spray volume, and air-assisted application strategies on deposition of pesticides in soybean canopy," Transactions of the ASABE, vol. 51, no. 5, pp. 1529-1537, 2008.

[11] Z. Lou, F. Xin, X. Han, Y. Lan, T. Duan, and W. Fu, "Effect of unmanned aerial vehicle flight height on droplet distribution, drift and control of cotton aphids and spider mites," Agronomy, vol. 8, no. 9, p. 187, 2018.

[12] W. Qin, X. Xue, S. Zhang, W. Gu, and B. Wang, "Droplet deposition and efficiency of fungicides sprayed with small uav against wheat powdery mildew," International Journal of Agricultural and Biological Engineering, vol. 11, no. 2, pp. 27-32, 2018.

[13] M. Lv, S. Xiao, T. Yu, and Y. He, "Influence of uav flight speed on droplet deposition characteristics with the application of infrared thermal imaging," International Journal of Agricultural and Biological Engineering, vol. 12, no. 3, pp. 10-17, 2019.

[14] B. Qiu, L. Wang, D. Cai, J. Wu, G. Ding, and X. Guan, "Effects of flight altitude and speed of unmanned helicopter on spray deposition uniform," Transactions of the Chinese Society of Agricultural Engineering, vol. 29, no. 24, pp. 25-32, 2013.

[15] S. Chen, Y. Lan, J. Li, Z. Zhou, J. Jin, and A. Liu, "Effect of spray parameters of small unmanned helicopter on distribution regularity of droplet deposition in hybrid rice canopy," Transactions of the Chinese Society of Agricultural Engineering, vol. 32 , no. 17 , pp. 40-46, 2016.

[16] G. Wang, Y. Lan, H. Qi, P. Chen, A. Hewitt, and Y. Han, "Field evaluation of an unmanned aerial vehicle (uav) sprayer: 
effect of spray volume on deposition and the control of pests and disease in wheat," Pest management science, vol. 75, no. 6, pp. $1546-1555,2019$.

[17] C. Wang, X. He, X. Wang, Z. Wang, S. Wang, L. Li, Z. Wang, S. Mei, et al., "Distribution characteristics of pesticide application droplets deposition of unmanned aerial vehicle based on testing method of deposition quality balance," Transactions of the Chinese Society of Agricultural Engineering, vol. 32, no. 24, pp. 89-97, 2016.

[18] T. Qing, Z. Ruirui, C. Liping, X. Min, Y. Tongchuan, and Z. Bin, "Droplets movement and deposition of an eight-rotor agricultural uav in downwash flow field," International Journal of Agricultural and Biological Engineering, vol. 10, no. 3, pp. $47-56,2017$.

[19] S. Chen, Y. Lan, J. Li, Z. Zhou, A. Liu, and Y. Mao, "Effect of wind field below unmanned helicopter on droplet deposition distribution of aerial spraying," International Journal of Agricultural and Biological Engineering, vol. 10, no. 3, pp. 67-77, 2017.

[20] S. Chen, Y. Lan, Z. Zhou, J. Liao, Q. Zhu, et al., "Effects of spraying parameters of small plant protection uav on droplets deposition distribution in citrus canopy.," Journal of South China Agricultural University, vol. 38, no. 5, pp. 97-102, 2017.

[21] P. Zang, Q. Lyu, S. Yi, S. He, Y. Liu, H. Pan, L. Deng, et al., "Evaluation of spraying effect using small unmanned aerial vehicle (uav) in citrus orchard," in The Sixth Asian Conference on Precision Agriculture, pp. 46-46, Chinese Society of Agricultural Engineering, 2015.

[22] Y. Tang, C. Hou, S. Luo, J. Lin, Z. Yang, and W. Huang, "Effects of operation height and tree shape on droplet deposition in citrus trees using an unmanned aerial vehicle," Computers and electronics in agriculture, vol. 148, pp. 1-7, 2018.

[23] P. T. Damos and M. Savopoulou-Soultani, "Development and statistical evaluation of models in forecasting moth phenology of major lepidopterous peach pest complex for integrated pest management programs," Crop Protection, vol. 29, no. 10, pp. 1190-1199, 2010.

[24] B. R. Blaauw, D. Polk, and A. L. Nielsen, "Ipm-cpr for peaches: incorporating behaviorally-based methods to manage halyomorpha halys and key pests in peach," Pest management science, vol. 71, no. 11, pp. 1513-1522, 2015.

[25] W. Shilin, S. Jianli, H. Xiongkui, S. Le, W. Xiaonan, W. Changling, W. Zhichong, and L. Yun, "Performances evaluation of four typical unmanned aerial vehicles used for pesticide application in china," International Journal of Agricultural and Biological Engineering, vol. 10, no. 4, pp. 22-31, 2017.

[26] Y. Meng, Y. Lan, G. Mei, Y. Guo, J. Song, and Z. Wang, "Effect of aerial spray adjuvant applying on the efficiency of 
small unmanned aerial vehicle for wheat aphids control," International Journal of Agricultural and Biological Engineering, vol. 11, no. 5, pp. 46-53, 2018.

[27] H. Zhu, M. Salyani, and R. D. Fox, "A portable scanning system for evaluation of spray deposit distribution," Computers and Electronics in Agriculture, vol. 76, no. 1, pp. 38-43, 2011.

[28] N. M. Razali, Y. B. Wah, et al., "Power comparisons of shapiro-wilk, kolmogorov-smirnov, lilliefors and anderson-darling tests," Journal of statistical modeling and analytics, vol. 2, no. 1, pp. 21-33, 2011.

[29] R. C. Blair and J. J. Higgins, "A comparison of the power of wilcoxon's rank-sum statistic to that of student's t statistic under various nonnormal distributions," Journal of Educational Statistics, vol. 5, no. 4, pp. 309-335, 1980.

[30] D. Smith, "Uniformity and recovery of broadcast sprays using fan nozzles," Transactions of the ASAE, vol. 35, no. 1, pp. 39-44, 1992.

[31] D. C. Montgomery, G. C. Runger, and N. F. Hubele, Engineering statistics. John Wiley \& Sons, 2009. 